Check for updates

Cite this: RSC Adv., 2018, 8, 26696

\title{
Rhamnazin attenuates inflammation and inhibits alkali burn-induced corneal neovascularization in rats
}

\author{
Yao Yu, $\dagger^{\mathrm{ab}}$ Xue-Zhi Zhou, $\dagger^{\mathrm{c}}$ Lei Ye, ${ }^{\mathrm{a}}$ Qing Yuan, ${ }^{a}$ Shelby Freeberg, ${ }^{\mathrm{d}} \mathrm{Ce}$ Shi, ${ }^{\mathrm{e}}$ \\ Pei-Wen Zhu, ${ }^{a}$ Jing Bao, ${ }^{a}$ Nan Jiang ${ }^{a}$ and Yi Shao (D)*a
}

\begin{abstract}
The purpose of our study was to determine whether rhamnazin inhibits corneal neovascularization in the rat alkali burn model, and alleviates the inflammatory response of the cornea. Rhamnazin inhibited the proliferation of HUVEC cells in a dose-dependent manner, and it also inhibited the migration and luminal formation of HUVEC cells. $20 \mu \mathrm{M}$ rhamnazin eye drops were applied to an animal model of corneal alkali burn neovascularization 4 times a day for 14 days. The corneal neovascularization in the rhamnazin group was obviously less than that in the PBS control group. In the rhamnazin group, the inflammatory index of the cornea decreased gradually over time, whereas the inflammatory index of the PBS group decreased only slightly with time. The corneal CNV area in the PBS group was significantly larger than that in the rhamnazin group. The expression level of VEGF protein of the rhamnazin group was lower than that in the PBS group, and the expression level of PEDF was significantly higher than that of the PBS group. Rhamnazin downregulated the expression of VEGFR2 protein and decreased the expression levels of $\mathrm{p}$-STAT3, p-MAPK and p-Akt proteins. This study provides a new idea for the study of the molecular mechanism of corneal neovascularization.
\end{abstract}

Received 13th April 2018

Accepted 29th June 2018

DOI: $10.1039 / c 8 r a 03159 b$

rsc.li/rsc-advances therapeutic agents is a hot spot in the study of corneal neovascularization.

Rhamnazin, one of the polyphenolic compounds, is often extracted from Ginkgo biloba, Salix, sea buckthorn and other medicinal plants. It is similar to natural products of quercetin and present in the flowers, fruits and leaves of many other plants. ${ }^{1}$ Studies have found that rhamnazin helps in protecting cardiovasculature, antioxidation, and immune regulation. ${ }^{7}$ It has also been found to have antitumor, antiviral, antiinflammatory, and anti-allergic functions, as well as other biological activities. It is widely used in the treatment of all kinds of cardiovascular diseases and tumors, such as gastric cancer, cervical cancer, nasopharyngeal carcinoma and lung cancer. ${ }^{2-5}$ However, the research on rhamnazin is focused on its antitumor activity, and there is no study on its role in ocular diseases, especially in corneal neovascularization. The purpose of this study is to investigate the effect of rhamnazin on corneal neovascularization induced by alkali burn.

The alkali burn animal model, an inflammatory model, is an important method to study the pathogenesis and treatment of inflammatory neovascularization. ${ }^{8}$ This study performed an in vitro cell experiment to observe the effects of different concentrations of rhamnazin on HUVEC cell proliferation, migration and tube formation. Meanwhile, alkali burn neovascularization rat models were used to study the effect of rhamnazin on corneal neovascularization in the prevention and treatment of inflammation in rats. Through research on the mechanism of 
rhamnazin on corneal neovascularization and inflammation control, we expected to fundamentally solve the two key problems, i.e. the difficulties of corneal repair after operation and immune rejection reaction. It is a new way in the treatment of corneal blindness and inflammation. Through this study, we can not only explain the progression and pathological mechanism of corneal neovascularization, but also help find effective drugs for anti-angiogenesis and inflammation, explore its optimal concentration, and evaluate the drug effects and prognosis, therefore providing information for treatment of corneal allograft rejection. The successful development of this medicine will provide a good treatment for most ocular neovascular diseases, and become a breakthrough point of ophthalmology.

From the current limited research data and our previous work, we can foresee that research of rhamnazin in the field of inflammation will open up a new direction of medical research. So far rhamnazin's function and the relationship are not clear, making it necessary to study the gene expression regulation and biological function in detail. Therefore, it is of great significance and application prospect to carry out further and detailed analysis for a new drug for inhibiting inflammation. The blindness caused by keratitis corneal damage is a common disease in the process of Chinese industrialization. Inflammatory pathogenesis is extremely complex. Study of the mechanisms of keratitis provides an important theoretical basis for new drug targets, and is expected to solve several key problems of postoperative neovascularization and inflammation rejection reaction. It is a new way in the treatment of corneal blindness, and has wide application in clinics. In addition, these will provide the theoretical reference and valuable direction of clinical treatment on other inflammation and neovascularization-related diseases such as cancer, diabetes and rheumatoid arthritis.

\section{Materials and methods}

\section{Cell culture and rhamnazin preparation}

HUVEC cells were purchased from PromoCell (Heidelberg, Germany) and cultured in EBM2 medium containing $2 \%$ fetal bovine serum and endothelial cell growth supplement (PromoCell) at $37{ }^{\circ} \mathrm{C}$. Cell growth was observed by inverted microscope. The logarithmic phase endothelial cells were used during the experiment. Rhamnazin (98\%, Sigma-Aldrich, St. Louis, MO) was dissolved in DMSO to prepare required concentration.

\section{Cell viability assay}

Cell viability was examined by CCK-8 kits. Briefly, HUVEC cells grown in logarithmic phase were inoculated in 96 well plates at $37^{\circ} \mathrm{C}$, with a density of $5 \times 10^{3}$ cells per well. After the overnight incubation, a final concentration of $0.1 \mu \mathrm{M}, 0.5 \mu \mathrm{M}, 1$ $\mu \mathrm{M}, 2 \mu \mathrm{M}, 5 \mu \mathrm{M}, 10 \mu \mathrm{M}$ and $20 \mu \mathrm{M}$ rhamnazin were added to the medium. The control group was treated with culture medium. Each group contained five replicates. 72 hours later, $10 \mu \mathrm{l}$ cell proliferation and cytotoxicity test CCK-8 solution was added to each well and cultured for an additional 4 hours at $37^{\circ} \mathrm{C}$. Optic density at the wavelength of $450 \mathrm{~nm}$ was read by a microplate reader.

\section{Wound closure assay}

There were $1 \times 10^{5}$ HUVEC cells in the logarithmic growth phase per well, seeded in 24 gelatin-coated wells for overnight culture at $37{ }^{\circ} \mathrm{C}$. A $200 \mu \mathrm{l}$ sterile pipette tip was used to draw scratches in the vertical direction, resulting in the horizontal interval of $2 \mathrm{~mm}$ scratches. Cells were treated with PBS, $5 \mu \mathrm{M}$ and $20 \mu \mathrm{M}$ rhamnazin and photographed at $0 \mathrm{~h}, 12 \mathrm{~h}$ and $24 \mathrm{~h}$ time points. The experiment was repeated 3 times.

\section{Tube formation assay}

24-well plates were coated with Matrigel basement membrane matrix (BD, Bioscience) in 37 degrees for 1 hour, and 10000 HUVEC cells per well in growth factor-free EBM2 medium with $0.1 \%$ FBS were added. Cells were then incubated for 6 hours at $37^{\circ} \mathrm{C}$ with PBS, $5 \mu \mathrm{M}$ and rhamnazin $(20 \mu \mathrm{M})$. Tube structures formed in the cavity of the artificial basement membrane were photographed under the inverted microscope. At least 5 areas for each well were selected for photograph. The total length of the lumen was the average value of 5 random fields of view at $100 \times$ magnification. The HUVEC branched out structure was quantified by ImageJ software.

\section{Alkali-burned rat corneas and treatment}

We have obtained ethics approval from Nanchang Royo Biotech corporation animals ethics committee.

60 healthy adult male SD rats weighing $180-200 \mathrm{~g}$ were used in the study (Shanghai, Shilaike, Laboratory, Animal, Co, Ltd., Shanghai, China). General anesthesia was induced by intraperitoneal injection of pentobarbital $\left(40 \mathrm{mg} \mathrm{kg}^{-1}\right)$, then the right eye was used as the experimental eye, and the left eye as the control eye. Eye drops of tetracaine were used as corneal surface anesthetics. Standard $3.5 \mathrm{~mm}$ diameter single circular filter paper was soaked in $1 \mathrm{M}$ sodium hydroxide solution for $20 \mathrm{~s}$, and excess liquid was absorbed. Under the microscope, the filter paper was pasted on the right central corneal surface for 30 s. After removing the filter paper, eyes were immediately flushed with $10 \mathrm{ml}$ PBS solution.

After the establishment of the alkali burn model, the animals were randomly divided into two groups, with 30 rats in each group. One group received $10 \mu \mathrm{l}$ topical PBS eye drops after alkali burn, 4 times a day, continuously for 14 days. The other group was treated with $10 \mu \mathrm{l} 20 \mu \mathrm{M}$ rhamnazin dissolved in PBS eye drops, 4 times a day, continuously for 14 days. The eyes of the rats were observed and evaluated by slit lamp microscope at $0,1,4,7,10$ and 14 days after eye drops. Corneal neovascularization, inflammatory response, and corneal epithelial damage were evaluated. 14 days later, the animals were euthanized and the corneas were removed from the experimental animals and preserved in the $-80{ }^{\circ} \mathrm{C}$ refrigerator for later histological detection and protein extraction. 


\section{Slit-lamp microscopic observation}

After establishing the alkali burn rat model, the ocular surface was stained with $1 \%$ sodium fluorescein. The injury of corneal epithelium in rats was observed under a cobalt blue light slit lamp microscopy, and through the image-processing software for image acquisition and processing. The growth of corneal neovascularization was examined daily, and the lengths of new vessels (length of continuous and with few bending new vessels tangent of corneal vertical prevail) were measured. Calculation of the neovascularization area was based on the formula $S=C$ / $12 \times 3.1416 \times\left[r^{2}-(r-I)^{2}\right]$, where $S$ is corneal neovascularization growth area, $C$ is the circumference of corneal neovascularization network, $r$ is the corneal radius, and $I$ is the new blood vessel length. The inflammatory index was analyzed as previously described. ${ }^{32}$

\section{Histology analysis}

The rat eyes were fixed in 4\% paraformaldehyde, dehydrated by gradient alcohol, processed by xylene and embedded in paraffin. Serial sections were made along the anterior and posterior diameters of the eyeball, with a thickness of $6 \mu \mathrm{m}$ and placed on a slide pretreated with poly-lysine. After deparaffinization, slides were stained with conventional hematoxylin and eosin, and the image was acquired under light microscopy.

\section{Western blot assay}

The corneal tissue was lysed in ice-cold RIPA buffer with protease inhibitors, and homogenized by ultrasound. Protein concentration was determined by BCA method. The same amounts of protein were subjected to SDS-PAGE electrophoresis, and transferred to PVDF. The membrane was blocked with $2 \%$ BSA for $1 \mathrm{~h}$, and blotted with primary antibodies against p-VEGF2 (1:200; Santa Cruz, USA), t-VEGF2 (1:200; Santa Cruz, USA), STAT3 (1:200; Santa Cruz, USA), p-MAPK (1:200; Santa Cruz, USA), t-MAPK (1:200; Santa Cruz, USA), p-Akt (1:200; Santa Cruz, USA), t-Akt (1:200; Santa Cruz, USA), $\beta$-actin (1:10 000; Bio-Rad, Hercules, CA, USA), VEGF (1:200; Santa Cruz, USA) and PDGF (1:200; Santa Cruz, USA) overnight at $4{ }^{\circ} \mathrm{C}$. After washing with TBST, horseradish peroxidase-labeled secondary antibody (1:5000; Dako, Shanghai, China) was added and incubated for 1 hour at room temperature. After washing with TBST and incubating with ECL reagent, film was used for signal development. ImageJ software was used to scan the gray value of each band, with $\beta$-actin as the reference control. Each experiment was repeated 3 times.

\section{Immunofluorescence}

The expression of STAT3 in HUVEC cells under different intervention conditions and the expression of VEGF and PEDF in the cornea under different intervention conditions were detected according to the methods described previously. ${ }^{33}$ Primary antibodies used in cell experiments were the mouse anti human STAT3 antibody with a dilution ratio of $1: 300$, and the sheep anti mouse VEGF and PEDF antibodies, with dilution ratios of $1: 100$ and $1: 200$, respectively. The nucleus was stained with
Hoechst 33342, and the samples were mounted with antiquenching agents. Images were acquired under a fluorescence microscopy.

\section{Statistical analysis}

Images were processed with Image-Pro Plus 6 software. SPSS 19 software was used for statistical analysis. The comparison between the groups was conducted using the analysis of variance test. The inflammatory index and the area and length of the corneal neovascularization were analyzed using ANOVA followed by Bonferroni post hoc comparison. The LSD- $t$ test was used to determine differences between two groups, and all statistical tests were two-sided. $p<0.05$ was statistically significant.

\section{Result}

\section{Rhamnazin inhibits the growth of the HUVEC cells in a dose-} dependent manner

Fig. 1A is the structure of rhamnazin. The inhibitory effect of rhamnazin on HUVEC growth was tested by CCK-8 assay in eight treated groups with different concentrations $(0,0.1,0.5,1$, $2,5,10$ and $20 \mu \mathrm{M}$ ). The CCK-8 showed that the viability of HUVEC was significantly inhibited when the concentration of rhamnazin was higher than $5 \mu \mathrm{M}$ (Fig. 1D). With the increase of rhamnazin concentration, the inhibition of the HUVEC growth was more obvious. The effective concentration of rhamnazin in the rats was $20 \mu \mathrm{M}$ in our preliminary experiment, so the rhamnazin concentration used in following animal experiments was $20 \mu \mathrm{M}$.

\section{Rhamnazin inhibits the cell migration and tube formation in HUVEC cells}

To examine the role of rhamnazin in HUVEC cell migration, the HUVEC cells were treated with $1 \%$ BSA, $5 \mu \mathrm{M}$ rhamnazin and 20 $\mu \mathrm{M}$ rhamnazin. The scratch healing process was observed at three time points ( $0 \mathrm{~h}, 12 \mathrm{~h}$ and $24 \mathrm{~h}$ ) (Fig. 1B). The woundclosure assay showed that the scratch healing rate of the 20 $\mu \mathrm{M}$ rhamnazin-treated group was significantly lower than the $1 \%$ BSA-treated group and the $5 \mu \mathrm{M}$ rhamnazin-treated group at $12 \mathrm{~h}$ and $24 \mathrm{~h}$. The scratch healing rate of $5 \mu \mathrm{M}$ rhamnazin at $12 \mathrm{~h}$ was obviously lower than the $1 \%$ BSA-treated group. The scratch of the $1 \%$ BSA-treated group and the $5 \mu \mathrm{M}$ rhamnazintreated group were closed at $24 \mathrm{~h}$, but the scratch of the 20 $\mu \mathrm{M}$ rhamnazin-treated group was still not healed. The migration rate of the $1 \%$ BSA treatment group and the $20 \mu \mathrm{M}$ rhamnazin treatment group at $12 \mathrm{~h}$ were about $100 \%$ and $50 \%$, respectively (Fig. 1C).

HUVEC cells were treated with 1\% BSA, $5 \mu \mathrm{M}$ rhamnazin and $20 \mu \mathrm{M}$ rhamnazin, and the ability of tube formation was assessed by an in vitro angiogenesis assay. ${ }^{1}$ The result showed that the number of lumens formed by the $20 \mu \mathrm{M}$ rhamnazintreated HUVEC cells was significantly lower than that of the $1 \%$ BSA- and $5 \mu \mathrm{M}$ rhamnazin-treated cells (Fig. 1E). We also measured the tube network length of HUVEC cells treated by 20 $\mu \mathrm{M}$ rhamnazin, $5 \mu \mathrm{M}$ rhamnazin and 1\% BSA (Fig. 1F). The 
A

B

E

$\mathrm{Oh}$

$12 \mathrm{~h}$

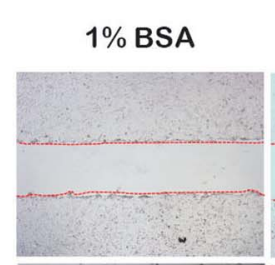

$$
5 \mu \mathrm{M}
$$
Rhamnazin

$24 \mathrm{~h}$
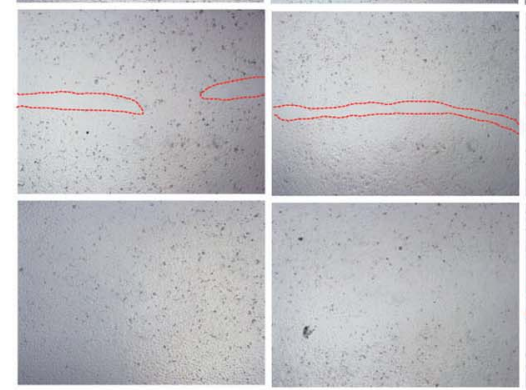

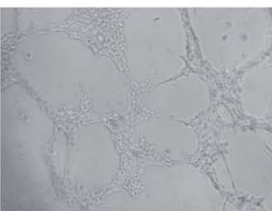

$1 \%$ BSA

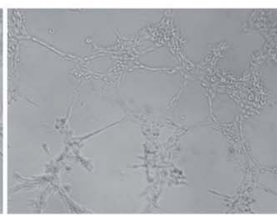

$5 \mu \mathrm{M}$ Rhamnazin

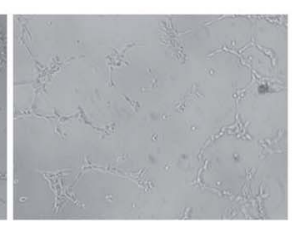

$20 \mu \mathrm{M}$
$20 \mu \mathrm{M}$

Rhamnazin

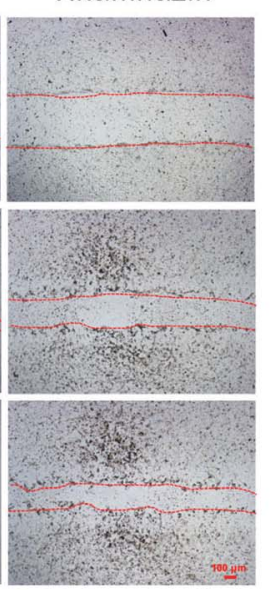

Rhamnazin
C

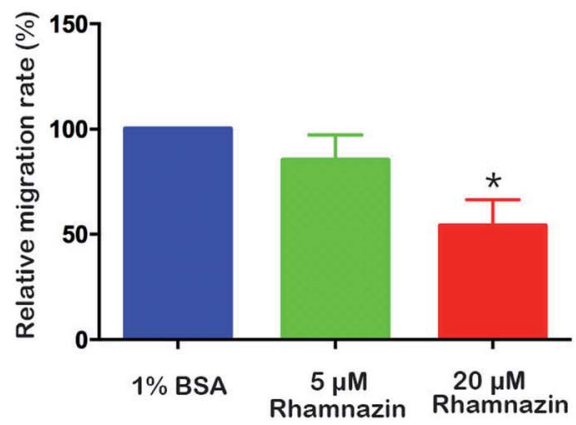

D

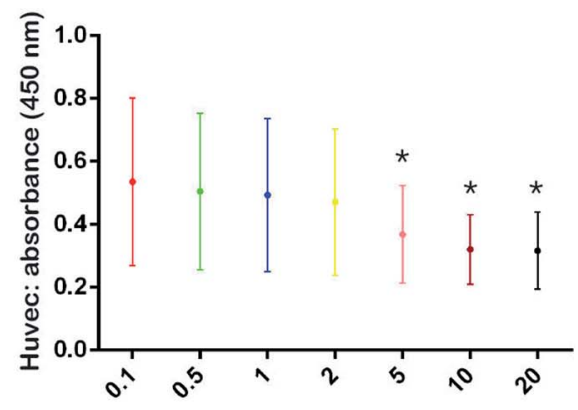

F

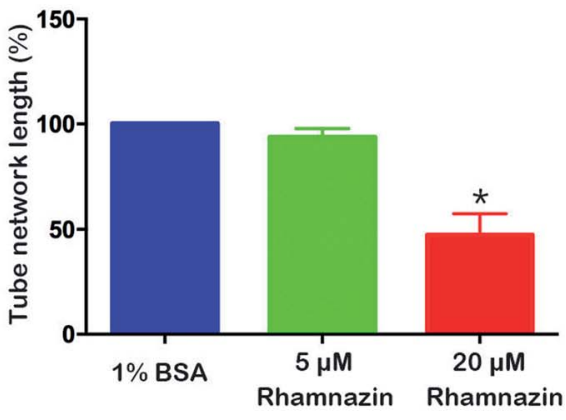

Fig. 1 Effects of rhamnazin on the proliferation, migration and tube formation of HUVEC. (A) The structure of rhamnazin. (B) Migration of HUVEC cultured in medium without serum in the control group, the $5 \mu \mathrm{M}$ rhamnazin group and the $20 \mu \mathrm{M}$ rhamnazin group at $0 \mathrm{~h}, 12 \mathrm{~h}$ and $24 \mathrm{~h}$. The effects of different concentrations of rhamnazin on HUVEC were accessed by CCK- 8 assay. (C) The relative migration rate of HUVEC in the control group, the $5 \mu \mathrm{M}$ rhamnazin group and the $20 \mu \mathrm{M}$ rhamnazin group were calculated at $12 \mathrm{~h}$. Results are expressed as percentage of wound closure (percentage closure) (mean \pm SD). (D) The effects of different concentration of rhamnazin on HUVEC were accessed by CCK- 8 assay. Each value represents the mean $\pm S D$, compared with the control group. (E) The HUVEC cells were pre-treated with EBM 2 medium for $12 \mathrm{~h}$. Tube structure of HUVEC grown in the control group and rhamnazin group was accessed after $1 \%$ BSA, $5 \mu M$ rhamnazin and $20 \mu \mathrm{M}$ rhamnazin were added in EBM2 medium for 6 h. (F) The tube network length rates were quantified by ImageJ. Data, and are presented as the mean $\pm S E$. $(n=3) * p$ $<0.05$ vs. control group, determined by one-way ANOVA test.

results suggest that $20 \mu \mathrm{M}$ rhamnazin blocked the tube formation compared with $1 \%$ BSA and $5 \mu \mathrm{M}$ rhamnazin in HUVEC cells.

\section{Metabolic condition of animal model}

In the $\mathrm{NaOH}$ induced alkali-burned Sprague Dawley rats (60 males) model, 60 rats were divided into two treatment groups randomly: a PBS-treated group and a $20 \mu \mathrm{M}$ rhamnazin-treated group (Fig. 2A) (4 times/1 day). We measured the body weight and eyeball weight of rats at 4, 7,10 and 14 days (Fig. 2B and C).
The results showed that there were no obvious differences between the PBS-treated group and the $20 \mu \mathrm{M}$ rhamnazintreated group in body weight and eyeball weight.

\section{Rhamnazin inhibited corneal neovascularization}

After the alkali burn model was completed, we observed the corneal neovascularization of rats in the rhamnazin-treated group and the PBS-treated group. The first day after the alkali burn, corneal neovascularization began to form at the limbus. The number of corneal neovascularizations at the central and 


\section{A}
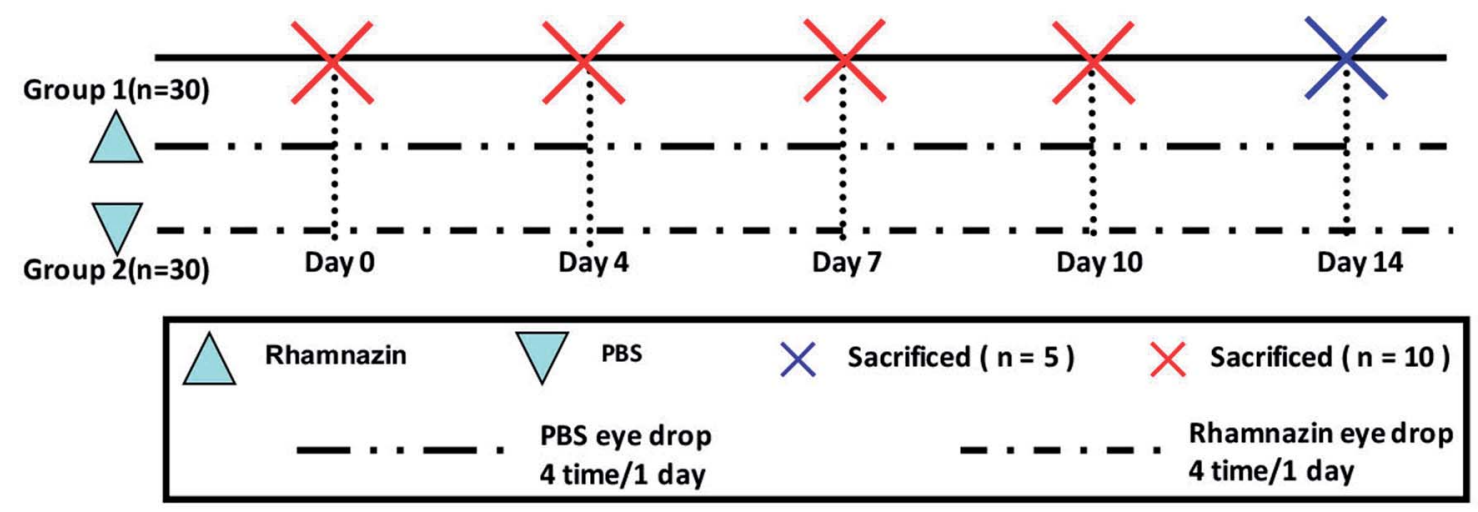

B
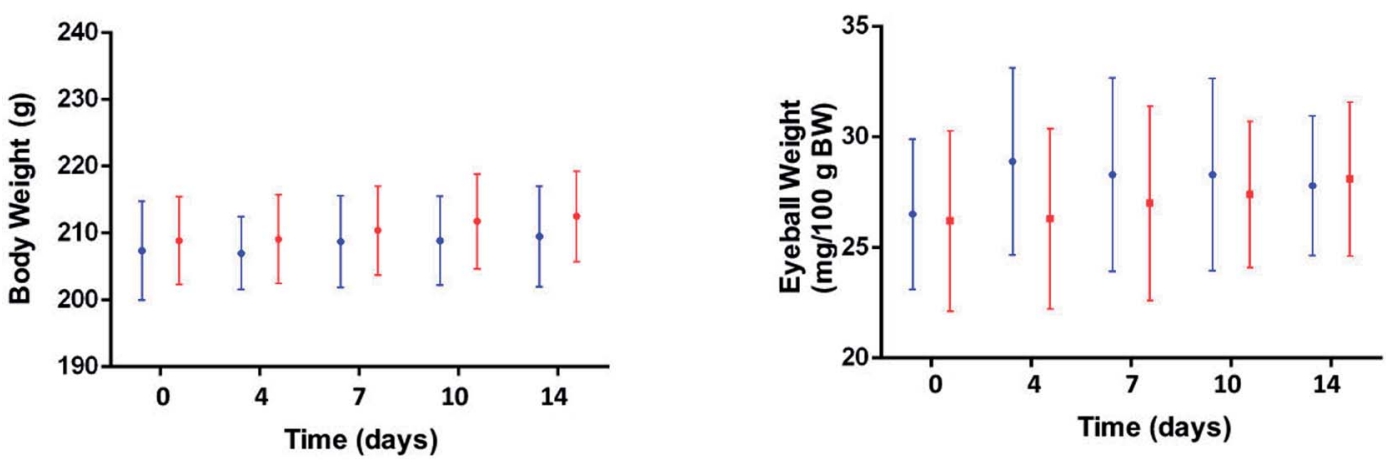

\section{$20 \mu M$ Rhamnazin $\square$ PBS}

Fig. 2 Metabolic conditions of animals in this research. (A) The experiment design of this study. 60 Sprague Dawley rats were used to establish corneal alkali burn models and divided into two groups randomly. In the control group, PBS was used in alkali burn corneal for 4 times per day. Rhamnazin was used in the experimental group for 4 times per day. (B) There was no significant difference in the body weight of Sprague Dawley rats between the control group and the rhamnazin group during the course of the experiment. (C) There was no obvious difference in eyeball weight between the control group and the rhamnazin group.

limbus in the rhamnazin-treated group was obviously less than that of the PBS-treated group at D14 (Fig. 3A). Histologic examination showed that treatment with PBS resulted in more neovascularization in the central corneal than treatment with rhamnazin at D14.

We also calculated the CNV area of the corneal at D0, D4, D7, D10 and D14. The CNV area in the PBS-treated group was significantly higher than the rhamnazin-treated group at the same point in time. The CNV area in the PBS-treated group was much higher than that of the rhamnazin-treated group at D7 and D14 (Fig. 3C). Over time, the CNV area of the PBS-treated group increased considerably, but the $\mathrm{CNV}$ area of the rhamnazin-treated group only showed subtle increase. The inflammatory index of the PBS-treated group increased in D7 and D10, while the inflammatory index decreased significantly from D1 to D14 in the rhamnazin-treated group. The inflammatory index of the PBS group was higher than the rhamnazin group at the same point in time (Fig. 3B).

\section{Rhamnazin regulates the expression of VEGF and PEDF}

IF detection of PEDF and VEGF expression was used to access the proangiogenic and antiangiogenic effects in the PBS group and the rhamnazin group at day 14 (Fig. 4A and B). VEGF staining in the rhamnazin group was infrequent, while the signal was obviously observed in the PBS group. PEDF staining was much higher in the rhamnazin group than the PBS group. We also evaluated the expression of VEGF and PEDF by western-blot (Fig. 4C and D). We found that VEGF protein levels in the PBS-treated group were higher than in the rhamnazin-treated group. Rhamnazin significantly upregulated PEDF expression at D14. Though the mechanism of rhamnazin is still unclear, our study suggested that rhamnazin regulated the expression of VEGF and PEDF in the corneal alkali burn model, suggesting rhamnazin may play a direct role in the inhibition of neovascularization. 
A

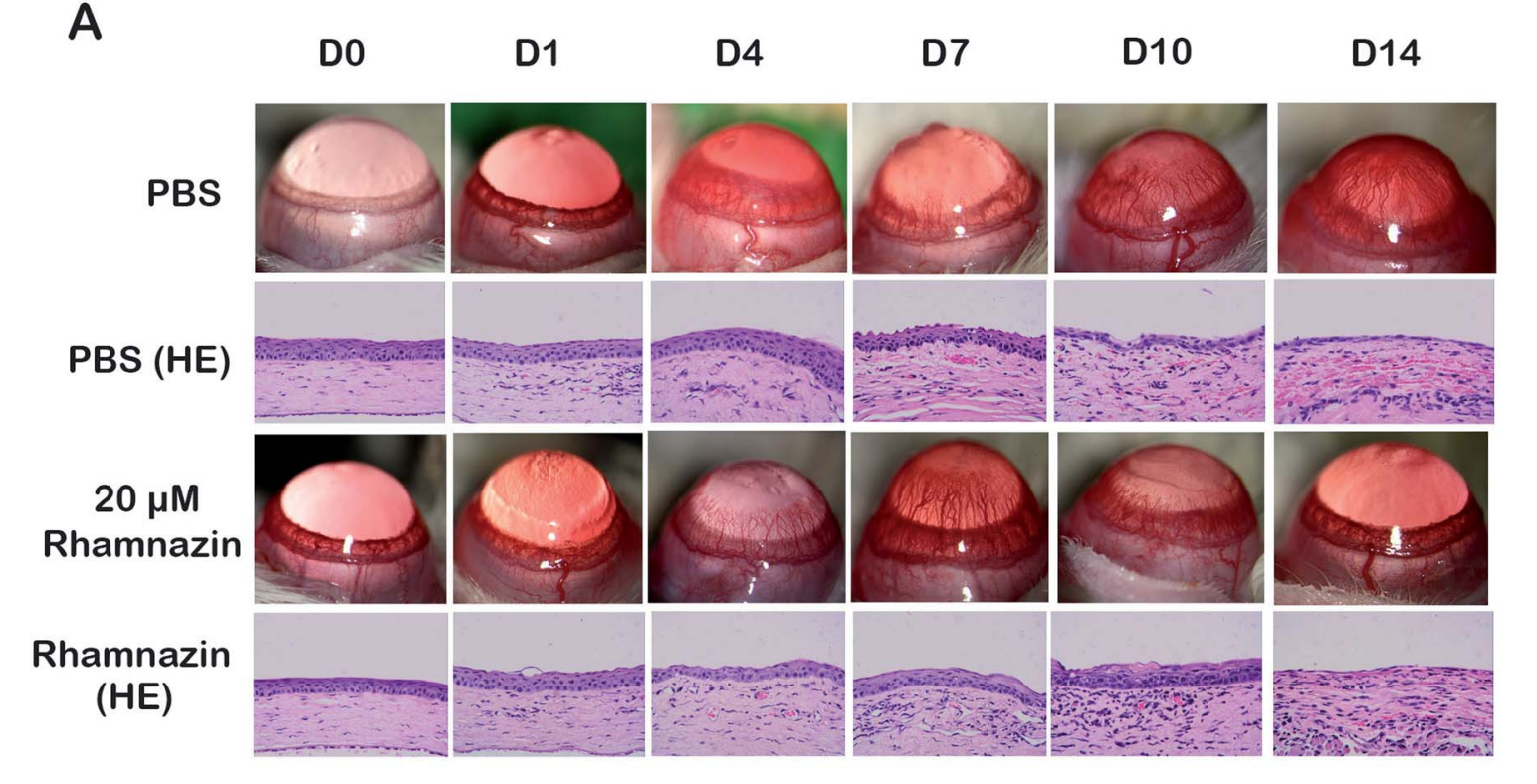

B

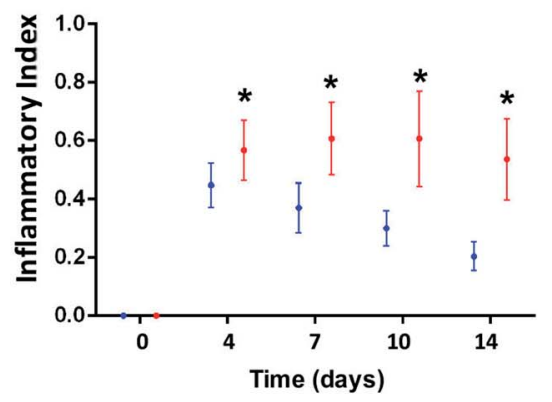

C

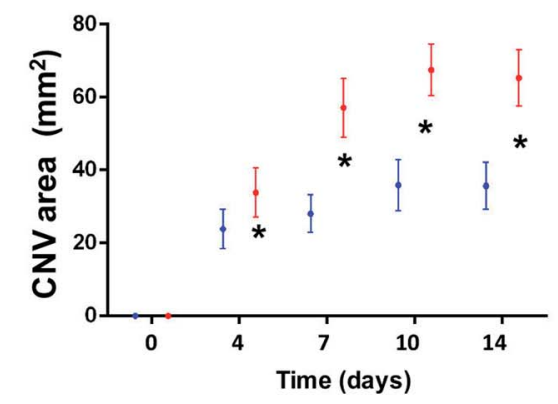

$20 \mu M$ Rhamnazin $\square$ PBS

Fig. 3 The effect of rhamnazin on corneal neovascularization in the corneal alkali burn model. (A) The corneal photography and histological examination of corneal alkali burn model treated with PBS and rhamnazin in 0,1, 4, 7, 10, 14 days. (B) Inflammatory index of corneal post-injury in PBS group and rhamnazin group on day $4,7,10,14(n=3, * p<0.05)$. (C) The corneal neovascularization area of the ocular surface was evaluated from day 1 to day 14 in both groups $(n=3, * p<0.05)$.

\section{Rhamnazin inhibits VEGFR2/STAT3 signal pathway}

In order to investigate the mechanism of inhibition of neovascularization of rhamnazin, we detected the expression level of VEGFR2-related signaling pathways in HUVEC cells (Fig. 5A). HUVEC cells were cultured in the presence of VEGF. Western blot assay showed that the p-VEGFR2 protein content in HUVEC cells treated with $20 \mu \mathrm{M}$ rhamnazin was obviously decreased compared with PBS treatment group and negative control group (Fig. 5B). To understand the signaling cascade initiated by VEGFR2, we also assessed the expression of pSTAT3, p-MAPK and p-Akt (Fig. 5A and C-F). Rhamnazin down-regulated the expression of p-STAT3 in VEGF-treated HUVEC cells. We also found that p-MAPK was robustly decreased in rhamnazin-treated HUVEC cells. The expression of p-Akt was significantly down-regulated by rhamnazin in VEGF cultured HUVEC cells. These data suggested that the down-regulation of the VEGFR2/STAT3/MAPK/Akt signal pathway by rhamnazin plays an important role in inhibiting angiogenesis in HUVEC cells (Fig. 6).

Subcellular localization of STAT3 activity is closely related to its activity, so the immunofluorescence and confocal imaging were used to access subcellular localization of STAT3 in VEGFtreated cultured HUVEC cells (Fig. 5B). In the VEGF group, the fluorescence intensity of the STAT3 in HUVEC cell nuclei was much stronger than the VEGF + rhamnazin group. The nuclear transcription level of STAT3 in the VEGF + rhamnazin group was significantly less than the VEGF group. These results showed that rhamnazin inhibited angiogenesis by regulating the STAT3-dependent signaling pathway.

\section{Discussion}

Ocular alkali burn is one of the most difficult emergencies in ophthalmology. The study of its treatment has been the focus 
A

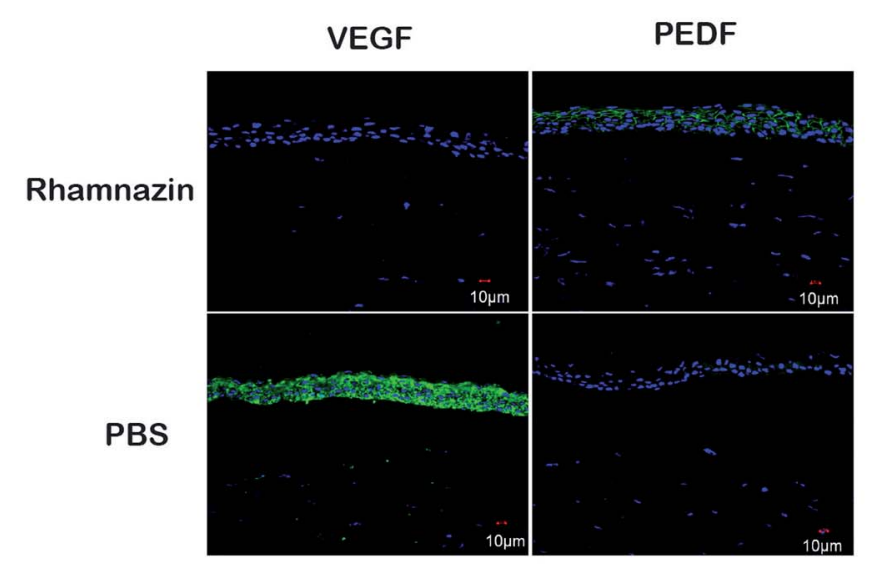

C

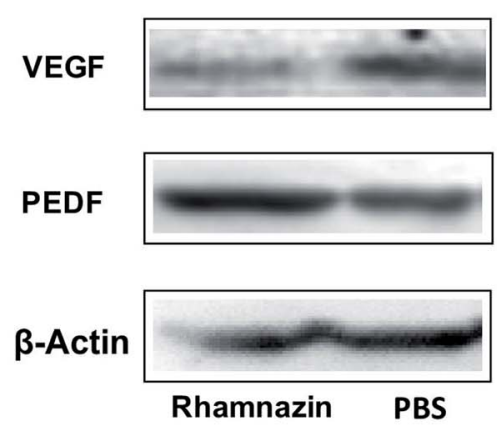

B

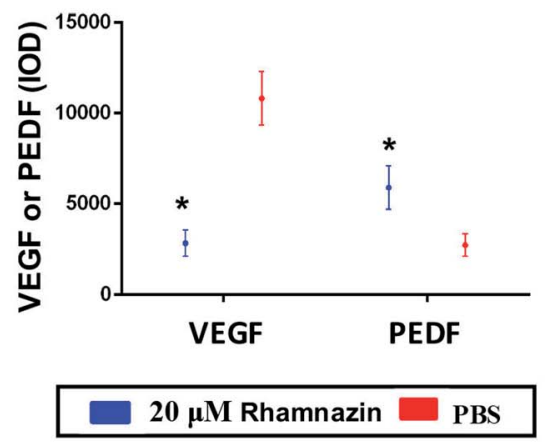

D

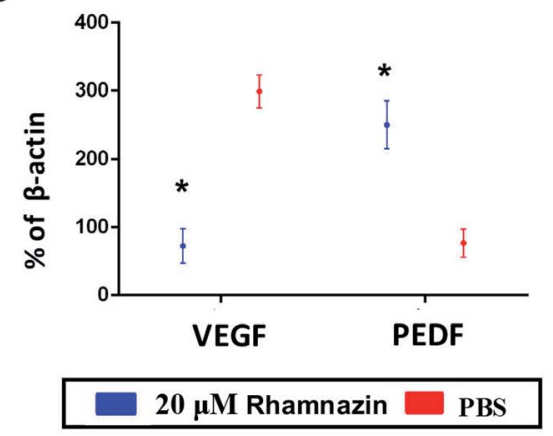

Fig. 4 The effects of rhamnazin on the expression of VEGF and PEDF in the corneal alkali burn model. (A) The VEGF and PEDF protein expression and location in the cornea at day 14 in the PBS group and rhamnazin were estimated by immunofluorescence assay. (B) Immunofluorescence intensity of VEGF and PEDF in two groups was quantified by ImageJ ( $n=3,{ }^{*} p<0.05$ ). (C) Western blot assay was used to access the protein expression of VEGF and PEDF in alkali burn cornea treated with PBS and rhamnazin. (D) Protein expression levels of VEGF and PEDF in cornea treated with PBS and rhamnazin was quantified by ImageJ $(n=3, * p<0.05)$.

and difficulty of ophthalmology. Ocular alkali burn results in the damage of ocular surface tissue, which can lead to epithelial necrosis, exfoliation, corneal perforation, corneal neovascularization, and so on. ${ }^{9,29,31}$ Controlling the inflammation, stabilizing the ocular surface, and inhibiting corneal neovascularization are key to the recovery of visual acuity in patients with corneal alkali burn. ${ }^{10,28}$ Neovascularization is the result of imbalance between factors that inhibit and promote angiogenesis after corneal injury. ${ }^{11,30}$ With the further development of neovascularization, it has been shown that VEGF plays a key role in neovascularization..$^{12}$ The study of the mechanism of corneal neovascularization and the drugs to inhibit angiogenesis are of great significance for the treatment of corneal neovascularization.

In this experiment, corneal alkali burn model is a representative model of ocular neovascularization and inflammatory diseases. It can lead to corneal ulceration, severe keratitis, corneal neovascularization, and formation of corneal scars. Therefore, it is widely used to study the mechanism and treatment of corneal inflammation and angiogenesis. In recent years, it has been discovered that rhamnazin has a series of pharmacological properties, including antioxidation and antitumor functions, but its mechanism of anti-angiogenesis has not been clearly explained. ${ }^{4-6}$ In the present study, we used the animal model to investigate the effects of rhamnazin on keratitis and neovascularization. For this reason, we treated corneas of alkali burn rats with $20 \mu \mathrm{M}$ rhamnazin. The results showed that the index of corneal inflammation and the area of neovascularization in the rhamnazin-treated group were smaller than those in the PBS group. The findings suggested that rhamnazin indeed inhibits corneal neovascularization, reduces corneal inflammation and promotes corneal epithelial repair. In vitro experimental results showed that rhamnazin can not only inhibit the proliferation of HUVEC cells, but also suppress HUVEC cell migration and tube formation. Therefore, we have shown that rhamnazin can inhibit the formation of corneal neovascularization both in vitro and in vivo.

Rhamnazin can inhibit corneal neovascularization, but its specific mechanism has not been elucidated. Studies have pointed out that the mechanism of anti-angiogenesis by rhamnazin is mainly through the vascular endothelial growth factor receptor (VEGFR). ${ }^{6}$ Vascular endothelial cell growth factor receptor (VEGFR) belongs to tyrosine kinase family proteins. There are four VEGF receptors: VEGFR-1 (Flt-1), VEGFR-2 (Flk-1/ 
A
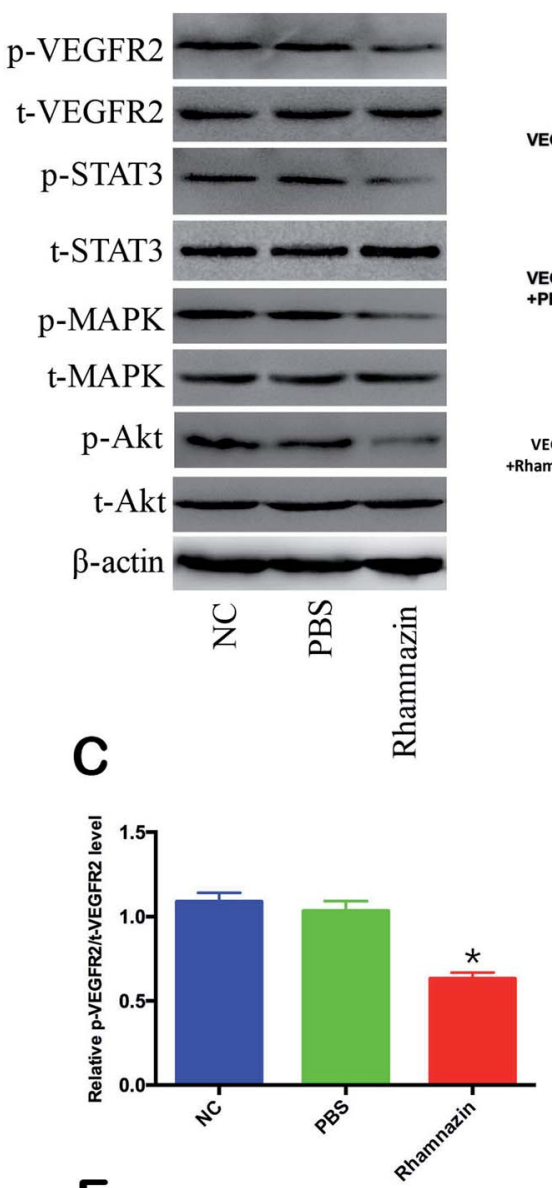

$\mathbf{E}$

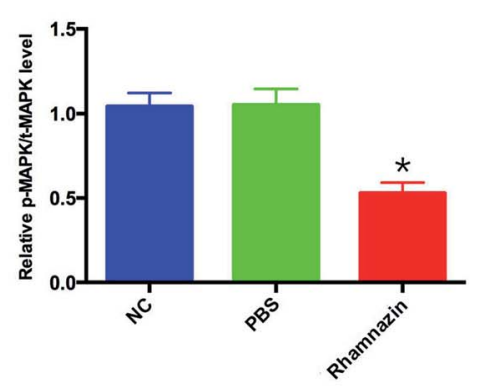

B

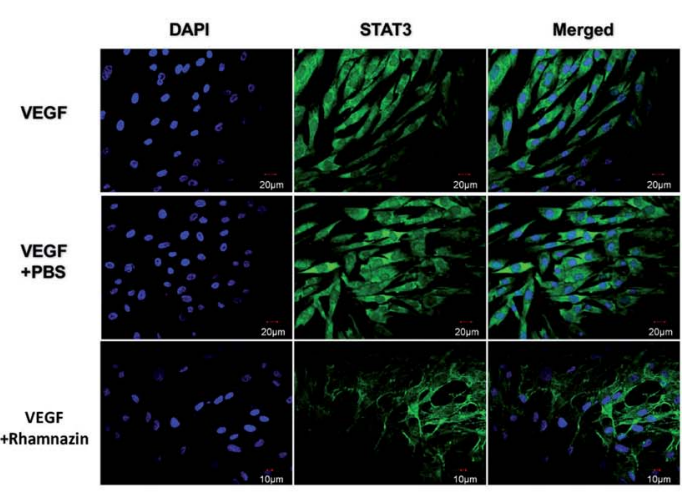

D
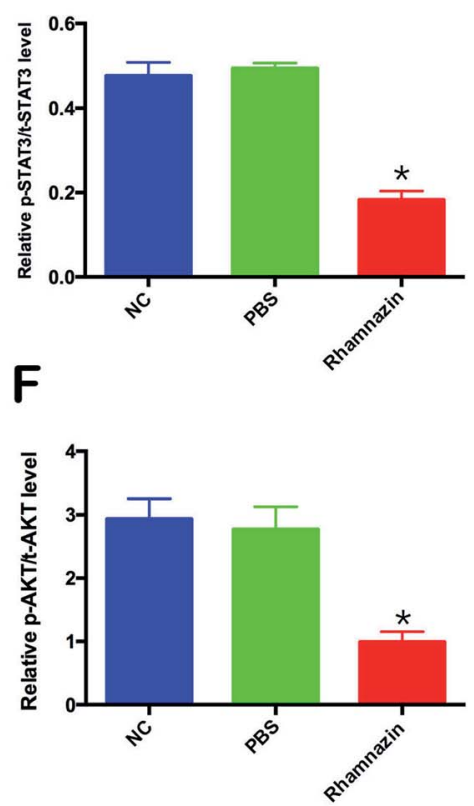

Fig. 5 The VEGFR2/STAT3/MAPK/Akt signal pathway was activated by rhamnazin in VEGF-stimulated HUVEC cells. (A) Representative western blot bands of p-VEGFR2, t-VEGFR2, p-STAT3, t-STAT3, p-MAPK, t-MAPK, p-Akt and t-Akt following treatment with PBS and $20 \mu M$ rhamnazin in VEGF-stimulated HUVEC cells for $24 \mathrm{~h}$. (B) Representative images of VEGF-stimulated HUVEC cells after $24 \mathrm{~h}$ of incubation with PBS or $20 \mu \mathrm{M}$ rhamnazin followed by IF staining for STAT3 (green) and DAPI (blue). Scale bar is $20 \mu \mathrm{m}$. (C-F) Quantitative analysis of $p$-VEGFR2, t-VEGFR2, $p$ STAT3, t-STAT3, p-MAPK, t-MAPK, p-Akt and t-Akt in VEGF-stimulated HUVEC cells of the control group, the PBS group and the rhamnazin group $(n=3, * p<0.05)$.

KDR), VEGFR-3 (Flt-4) and VEGFR (neuropilin-1). ${ }^{\mathbf{1 3 , 1 4}}$ VEGFR-1 and VEGFR-2 are mainly expressed in vascular endothelial cells, whereas VEGFR-3 is mainly expressed in lymphocytes. ${ }^{\mathbf{1 5}}$ After the invasion of new blood vessels, at least six different angiogenic-related growth factors are secreted, among which VEGF is the most important angiogenic factor. ${ }^{16}$ The specific effects of VEGF on vascular endothelial cells are achieved by two types of receptor tyrosine kinase (RTK) regulatory families,
VEGFR1 and VEGFR2. ${ }^{17}$ Sugimachi K. and other studies have found that the expression rate of VEGFR2 in tumor regions is $100 \%$, and the expression of VEGFR1 is not different from normal cells, which further proves that VEGFR2 is closely related to neovascularization. ${ }^{18}$ Recent studies have shown that when Notch or VEGFR3 is absent, a small amount of VEGFR2 can also form inflammatory neovascularization, while VEGFR2 alone/combined with VEGFR3 upregulates endothelial cell 


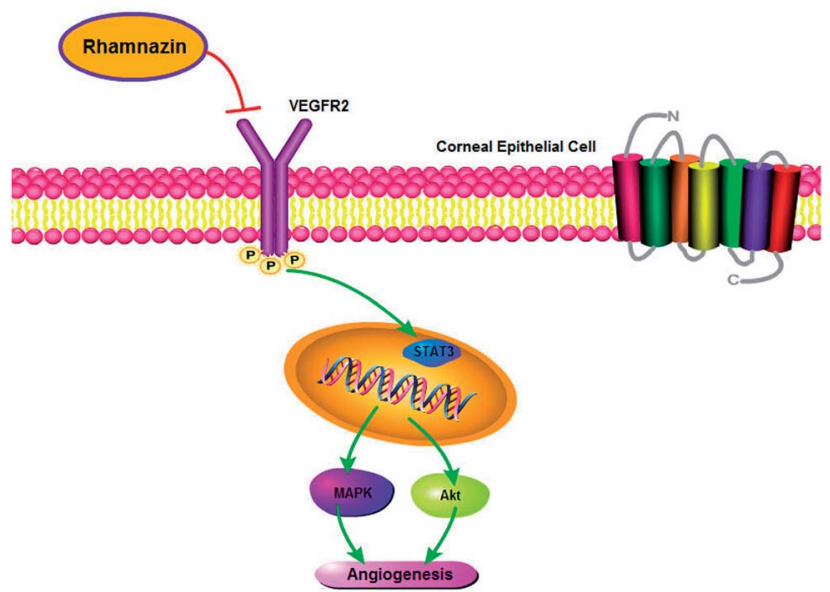

Fig. 6 The mechanism of rhamnazin-induced inhibition of angiogenesis in corneal epithelial cell. Rhamnazin inhibits corneal neovascularization by down-regulation of the VEGFR2/STAT3/MAPK/Akt signal pathway.

DLL4 and promotes neovascularization. ${ }^{19}$ Fengyun Dong found that DHA can downregulate the expression of VEGFR2 cells to inhibit the proliferation and migration of HUVECs. ${ }^{20}$ In order to investigate whether rhamnazin inhibits corneal neovascularization through VEGF and its receptor VEGFR2, expression levels of VEGF and VEGFR2 were detected by western-blot. The results showed that the expression levels of VEGF and p-VEGFR2 in the cornea of the PBS-treated group were significantly higher than that in the rhamnazin treatment group, and the expression level of the two proteins decreased significantly in the rhamnazin treatment group. We also demonstrated by immunofluorescence that the expression of VEGF in the cornea was lower in the rhamnazin-treated group than in the PBS group. These results confirm our hypothesis that rhamnazin inhibits corneal neovascularization by downregulating the expression of VEGF and its receptor VEGFR2.

The development of new blood vessels is mediated by a complex array of cellular and molecular factors. VEGF and PEDF play important roles in the development of new blood vessels. PEDF is an effective endogenous anti-angiogenic factor, highly expressed in the cornea. Studies have shown that the exogenous expression of PEDF inhibitors in corneal stroma leads to corneal neovascularization. ${ }^{21}$ However, VEGF plays an important role in promoting angiogenesis and promoting the development of inflammation. Studies have shown that the expression of VEGF in vascular endothelial cells is significantly higher in inflammatory and neovascularization corneas. $^{22}$ Therefore, breaking the balance between VEGF and PEDF may be the pathological mechanism of the development of corneal neovascularization. ${ }^{23}$ In our study, the expression of VEGF in the alkali burned cornea was significantly increased and the expression of PEDF was significantly decreased compared to the PBS control group. However, in the $20 \mu \mathrm{M}$ rhamnazin treatment group, the expression of VEGF in the corneal tissue of the alkali burned rats was significantly downregulated, while the expression of PEDF was significantly up-regulated. Thus, rhamnazin reestablished the balance between the VEGF and PEDF, thereby inhibiting the formation of new blood vessels caused by alkali burn.

VEGFR2 can efficiently activate multiple downstream signaling components, including mitogen activated protein kinase (MAPK), phosphorylation of serine/threonine kinase (Akt), signal transducer and activator of transcription factor 3 (STAT3), therefore promoting the growth of tumors and vascular endothelial cell proliferation, migration and tube formation. ${ }^{24-26}$ Previous studies have shown that inflammation is closely related to angiogenesis and lymphangiogenesis. VEGFR2 controls angiogenesis and lymphangiogenesis, and mediates multiple cell signaling pathways involved in inflammatory reaction. $\mathrm{X}$. Zhang showed that the indirubin can inhibit the JAK/STAT3 signal pathway in VEGFR2 mediated tumor vascular inflammation model, by inhibiting the migration and the formation of vascular endothelial cells and the release of inflammatory mediators. ${ }^{27}$ Therefore, we hypothesized whether rhamnazin can inhibit the neovascularization by regulating the downstream factors associated with VEGFR2. To confirm this hypothesis, we examined the expression levels of VEGFR2, STAT3, MAPK, and Akt in VEGF-treated HUVEC cells under different intervention conditions. The results showed that the protein expression levels of p-VEGFR2, p-STAT3, p-MAPK and p-Akt in the rhamnazin treatment group were significantly lower than those in the control group. Immunofluorescence showed reduced levels of STAT3 in the nucleus of VEGF-treated HUVEC cells in the rhamnazin group. These results confirmed that the mechanism of inhibition of neovascularization by rhamnazin is through the inhibition of VEGFR2 and its downstream STAT3/MAPK/Akt signaling pathway.

Overall, this study demonstrated that local administration of rhamnazin could inhibit inflammation and neovascularization after alkaline burn. Its anti-inflammatory and anti-angiogenesis effects are achieved by regulating the STAT3/ MAPK/Akt signaling pathway via VEGFR2 protein. Therefore, rhamnazin is a potential drug for the treatment of keratitis and neovascularization.

\section{Conflicts of interest}

None.

\section{Acknowledgements}

This work was supported by grants from the National Natural Science Foundation of China (NSFC No. 81160118 [Yi Sh], 81460092 [Yi Sh], 81460372 [Yi Sh] and 81660152 [Yi Sh]), Youth Science Foundation of Jiangxi Province (No. 20161BAB215198 [Yao Y.], 20151BAB215016 [Yi Sh]), Health Commission Foundation of Jiangxi Province (No. 20164017 [Yao Y.]), Key Education Department Foundation of Jiangxi Province (No. GJJ160020 [Yi Sh]). 


\section{References}

1 S. Resnikoff, D. Pascolini, D. Etya'ale, I. Kocur, R. Pararajasegaram, et al., Global data on visual impairment in the year 2002, Bull. W. H. O., 2004, 82, 844851.

2 X. W. Gao, Y. Fu, W. J. Li, A. J. Du, X. Li and X. D. Zhao, Mechanism of immune tolerance induced by donor derived immature dendritic cells in rat high-risk corneal transplantation, Int. J. Ophthalmol., 2013, 6, 269-275.

3 H. Cai, Z. Xie, G. Liu, et al., Isolation, identification and activities of natural antioxidants from Callicarpa kwangtungensis Chun, PLoS One, 2014, 9, e93000.

4 B. Ryu, H. M. Kim, J. S. Lee, et al., New Flavonol Glucuronides from the Flower Buds of Syzygium aromaticum (Clove), J. Agric. Food Chem., 2016, 64, 30483053.

5 A. A. Philchenkov and M. P. Zavelevych, Rhamnazin Inhibits Proliferation and Induces Apoptosis of Human Jurkat Leukemia Cells in Vitro, Ukr. Biochem. J., 2015, 87, 122-128.

6 Y. Yu, W. Cai, C. G. Pei and Y. Shao, Rhamnazin, a novel inhibitor of VEGFR2 signaling with potent antiangiogenic activity and antitumor efficacy, Biochem. Biophys. Res. Commun., 2015, 458, 913-919.

7 N. D. Martini, D. R. Katerere and J. N. Eloff, Biological activity of five antibacterial flavonoids from Combretum erythrophyllum (Combretaceae), J. Ethnopharmacol., 2004, 93, 207-212.

8 R. Cheng, C. Li, C. Li, et al., The artemisinin derivative artesunate inhibits corneal neovascularization by inducing ROS-dependent apoptosis in vascular endothelial cells, Invest. Ophthalmol. Visual Sci., 2013, 54, 3400-3409.

9 J. W. Kim, H. Jeong, M. S. Yang, C. W. Lim and B. Kim, Therapeutic effects of zerumbone in an alkali-burned corneal wound healing model, Int. Immunopharmacol, 2017, 48, 126-134.

10 S. Barrientos, O. Stojadinovic, M. S. Golinko, H. Brem and M. Tomic-Canic, Growth factors and cytokines in wound healing, Wound Repair Regen., 2008, 16, 585-601.

11 C. C. Hsu, H. M. Chang, T. C. Lin, et al., Corneal neovascularization and contemporary antiangiogenic therapeutics, J. Chin. Med. Assoc., 2015, 78, 323-330.

12 J. H. Chang, N. K. Garg, E. Lunde, K. Y. Han, S. Jain and D. T. Azar, Corneal neovascularization: an anti-VEGF therapy review, Surv. Ophthalmol., 2012, 57, 415-429.

13 N. Ferrara, H. P. Gerber and J. LeCouter, The biology of VEGF and its receptors, Nat. Med., 2003, 9, 669-676.

$14 \mathrm{~S}$. Koch and L. Claesson-Welsh, Signal transduction by vascular endothelial growth factor receptors, Cold Spring Harbor Perspect. Med., 2012, 2, a006502.

15 M. Shibuya, Vascular endothelial growth factor and its receptor system: physiological functions in angiogenesis and pathological roles in various diseases, J. Biochem., 2013, 153, 13-19.

16 N. Ferrara, Vascular endothelial growth factor: basic science and clinical progress, Endocr. Rev., 2004, 25, 581-611.
17 A. Hoeben, B. Landuyt, M. S. Highley, H. Wildiers, A. T. Van Oosterom and E. A. De Bruijn, Vascular endothelial growth factor and angiogenesis, Pharmacol. Rev., 2004, 56, 549-580.

18 K. Sugimachi, S. Tanaka, T. Terashi, K. Taguchi, T. Rikimaru and K. Sugimachi, The mechanisms of angiogenesis in hepatocellular carcinoma: angiogenic switch during tumor progression, Surgery, 2002, 131, S135-S141.

19 C. K. Domigan and M. L. Iruela-Arispe, Recent advances in vascular development, Curr. Opin. Hematol., 2012, 19, 176183.

20 F. Dong, X. Zhou, C. Li, et al., Dihydroartemisinin targets VEGFR2 via the NF kappaB pathway in endothelial cells to inhibit angiogenesis, Cancer Biol. Ther., 2014, 15, 1479-1488.

21 S. I. Yeh, T. C. Ho, S. L. Chen, et al., Pigment EpithelialDerived Factor Peptide Regenerated Limbus Serves as Regeneration Source for Limbal Regeneration in Rabbit Limbal Deficiency, Invest. Ophthalmol. Visual Sci., 2016, 57, 2629-2636.

22 R. Y. Kim, S. K. Chung, M. S. Kim and H. Ra, Effects of Combined Photodynamic Therapy and Topical Bevacizumab Treatment on Corneal Neovascularization in Rabbits, Cornea, 2016, 35, 1615-1620.

23 K. Ohno-Matsui, I. Morita, J. Tombran-Tink, et al., Novel mechanism for age-related macular degeneration: an equilibrium shift between the angiogenesis factors VEGF and PEDF, J. Cell. Physiol., 2001, 189, 323-333.

24 Y. Liu, Y. Qiao, C. Hu, et al., VEGFR2 inhibition by RNA interference affects cell proliferation, migration, invasion, and response to radiation in Calu-1 cells, Clin. Transl. Oncol., 2016, 18, 212-219.

25 W. Wang, F. Ren, Q. Wu, D. Jiang, H. Li and H. Shi, MicroRNA-497 suppresses angiogenesis by targeting vascular endothelial growth factor A through the PI3K/AKT and MAPK/ERK pathways in ovarian cancer, Oncol. Rep., 2014, 32, 2127-2133.

26 J. Zhang, C. Liu, W. Shi, et al., The novel VEGF receptor 2 inhibitor YLL545 inhibits angiogenesis and growth in breast cancer, Oncotarget, 2016, 7, 41067-41080.

27 X. Zhang, Y. Song, Y. Wu, et al., Indirubin inhibits tumor growth by antitumor angiogenesis via blocking VEGFR2mediated JAK/STAT3 signaling in endothelial cell, Int. J. Cancer, 2011, 129, 2502-2511.

$28 \mathrm{~J}$. Yu, X. L. Zheng, Y. Y. Li, et al., Clinical findings associated with Bidens bipinnata L. eye drops on moderate and severe dry eye in postmenopausal women, Int. J. Clin. Exp. Med., 2016, 9, 5643-5654.

29 Y. Yu, J. Zou, Y. Han, et al., Effects of intravitreal injection of netrin-1 in retinal neovascularization of streptozotocininduced diabetic rats, Drug Des., Dev. Ther., 2015, 9, 63636377.

30 Y. Han, Y. Shao, Z. R. Lin, et al., Netrin-1 simultaneously suppresses corneal inflammation and neovascularization, Invest. Ophthalmol. Visual Sci., 2012, 53, 1285-1295.

31 Y. Shao, Y. Yu, C. Li, J. Yu, R. R. Zong and C. G. Pei, Synergistic effect of quercetin and 6-gingerol treatment in streptozotocin induced type 2 diabetic rats and poloxamer 
P-407 induced hyperlipidemia, RSC Adv., 2016, 6, 1223512242.

32 C. Laria, J. L. Alio and J. M. Ruiz-Moreno, Combined nonsteroidal therapy in experimental corneal injury, Am. $J$. Ophthalmol., 1997, 29, 145-153.
33 F. Cristina, R. Graham, L. C. Claire, B. Muhammad, A. T. Brian and H. Toin, Endothelial heparan sulfate 6-Osulfation levels regulate angiogenic responses of endothelial cells to broblast growth factor 2 and vascular endothelial growth factor, J. Biol. Chem., 2012, 287, 3613236146. 